Review Article

Archives of Disease in Childhood, 1972, 47, 163.

\title{
Investigation of Degenerative Disease of the Central Nervous System
}

\author{
JOHN WILSON \\ From The Hospital for Sick Children, Great Ormond Street, London
}

Degenerative diseases of the nervous system in children are acknowledged to be chronic (usually) and destructive (invariably), and for the biochemically oriented clinician they provide a challenging interest because most are genetically, and probably, therefore, chemically determined. Individually relatively rare, most of them are inherited as autosomal recessive conditions, and by implication may represent the clinical effects of inborn metabolic errors. Nevertheless only a minority at present are thus identified because, except where there is an easily recognizable storage product such as ganglioside, or abnormal metabolic cumulation readily identifiable in body fluids such as amino acid, it is usually very difficult to guide biochemical research on purely clinical and pathological indications.

It is still necessary and possible, however, to give an accurate prognosis and genetic analysis without any biochemical information, but biochemical refinements offer the opportunity of delineating syndromes which at a given point in time are clinically indistinguishable, e.g. Tay-Sachs variants, and this may be important in genetic counselling. In a few conditions biochemical identification of fetal tissues enables a more constructive opportunity for eugenic measures.

At the present stage of our knowledge, and with perennial economic limitations on screening measures, a discriminating use of elaborate biochemical techniques is desirable and it is proposed to review a scheme of investigation which depends for its usefulness upon a careful clinical appraisal, and therefore is applicable to conditions which are not identifiable chemically as well as those that are.

A broad neuropathological classification of heredodegenerative neurological diseases is presented in Table I.

\section{TABLE I}

Neuropathological Classification of Heredodegenerative Neurological Diseases

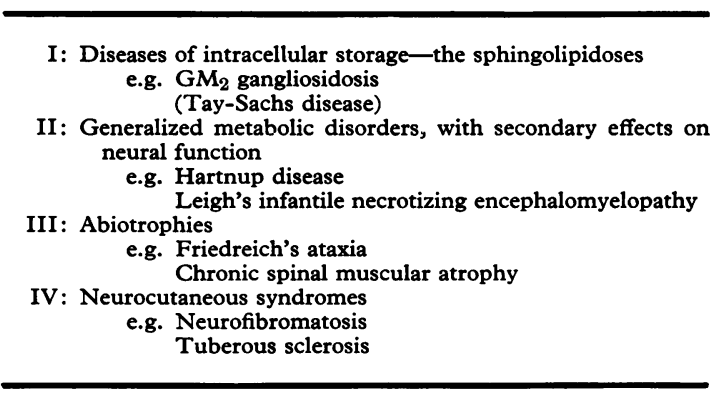

Abiotrophy is the term suggested by Gowers to represent conditions in which, after apparently normal differentiation and function, tissues degenerate and die. Recent usage by some workers has implied that tissues are prematurely effete, perhaps because of faulty differentiation or structure, but such a view is purely speculative.

Though the suggested classification is intended to be helpful in orienting investigations, the groups are not mutually exclusive. For example, though intracellular metabolic derangement in Group I may be primarily responsible for most of the symptoms, there may also be secondary generalized metabolic derangement, as has been suggested in, for example, Niemann-Pick disease. Moreover, the abiotrophic disorders may represent the effects of either intracellular or generalized accumulation of as yet unrecognized metabolites.

The diseases of intraneuronal storage have been intensively studied and the biochemical basis of many of them is now known and they are listed in 
Table II, with their 'chemical' as well as 'familiar' names.

\section{Clinical Appraisal}

Perhaps the most difficult clinical problem is to decide whether or not a condition is progressive, and yet this is usually the most important step in the diagnosis of a degenerative disease. In those situations where a previous child is affected by a recognized heredodegenerative disease it may be possible to obtain discriminatorily valuable findings from special laboratory studies some time before clinical abnormalities appear in a sib. For example, a rectal biopsy may show significant abnormalities and white cell hexosaminidase may be deficient in a homozygous newborn sib of a child with Tay-Sachs disease several months before its development becomes abnormal.

At the first meeting with a patient, without a prospective personal longitudinal analysis of a condition, one is entirely dependent on an evaluation of a retrospective narrative and, because of this, time is well spent on listening to a carefully elicited history. Unless it is then possible on examination and utilizing suitable laboratory investigations to establish a diagnosis, it may be necessary to wait until the evolving character of the condition declares itself on prospective review. The clinician's case notes (no matter how assiduously maintained) and memory (faithful-and failing) are most valuably supplemented by cine-film of gait or co-ordination, which may be the best way of disclosing the changing character of very slowly progressive diseases.

Dementia. Dementia is a characteristic early clinical feature of all the diseases of intraneuronal storage, and a common but later feature of other degenerative diseases, but is conspicuously absent, even preterminally, in conditions such as chronic spinal muscular atrophy. Dementia in infancy and early childhood manifests itself first as a slowing, then arrest, of acquisition of skills (linguistic or physical) before there is obvious regression. It is important to distinguish dementia from both primary mental subnormality and from psychotic regression (e.g. autism), but from a careful history

TABLE II

Nature of Biochemical Abnormalities in Some Sphingolipidoses

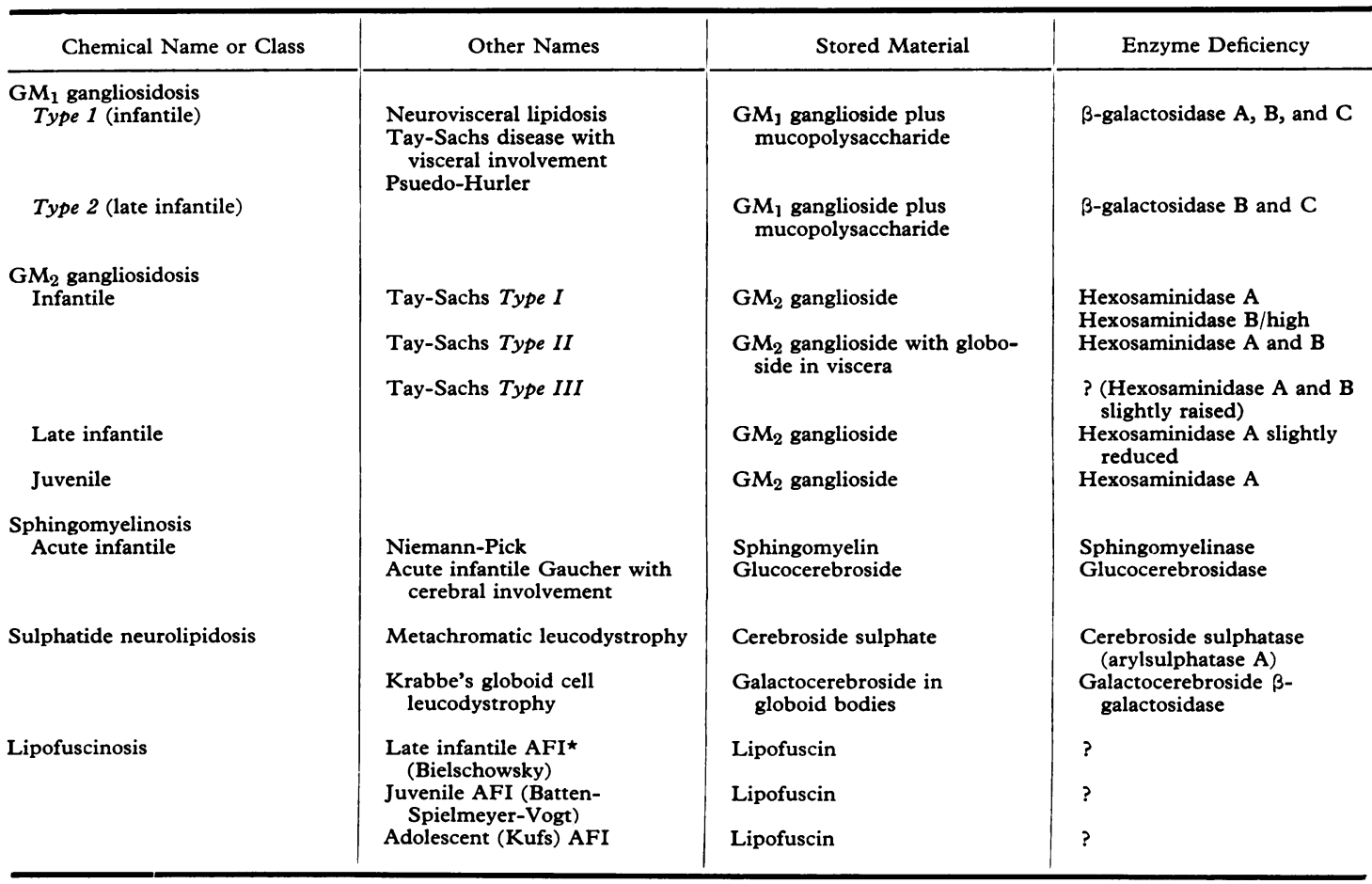

^Amaurotic family idiocy. 
supplemented by incidental documentation (baby books or photographs) it is usually possible to distinguish nonprogressive subnormality from more dynamic loss even in very young children in whom the tempo of degenerative disease tends to be more rapid than in older children. In older children deteriorating school performance, often associated with behaviour problems due to organic dementia (which is comparatively rare), may be exceedingly difficult to distinguish from the much more common psychosocial causes of failing academic performance and misbehaviour. Only longitudinal comparison over a few months will allow the distinction clinically, supplemented by serial psychological and usually EEG assessment.

Though in the more diffuse diseases no part of the neuraxis is spared, increasing weakness, ataxia, and clumsiness are the commonest manifestations of changing physical status, and these features are best recorded by cine-film.

Seizures. Seizures may be an early feature of a large number of degenerative diseases. They are particularly common in diseases primarily of grey matter, whereas in the leucodystrophies, if they occur at all, they develop relatively late in the natural history. Myoclonic attacks and 'minor status epilepticus' may be a distinctive feature of, for example, Tay-Sachs disease and late infantile (Bielschowsky) amaurotic family idiocy.

Before discussing the relevance of laboratory studies in evaluation of degenerative diseases, it is well to consider the differential diagnosis and the clinical pitfalls.

\section{Differential Diagnosis}

1. Tumour. The principal potentially sinister alternative cause of an evolving illness is a tumour, particularly of brainstem and posterior fossa. Posterior fossa tumours are especially frequent in childhood and their very early diagnosis may be very difficult when invasive rather than spaceoccupying characteristics present. A mixed picture of cerebellar and pyramidal dysfunction can be very misleading. If coexisting clinical abnormalities (such as cranial nerve palsies) do not give more positive clues, then air studies usually resolve the doubt. Exceptionally they may be normal.

2. Subacute sclerosing panencephalitis (SSPE). Although evidence from the United States and Canada suggests that the incidence of this condition is declining, perhaps as a result of widespread inoculation with attenuated measles virus, cases are still occurring in the United Kingdom.
Dementia may be a presenting finding, but fits usually occur early in the course of the disease. Not only are there major seizures often with focal features, but also there are 'hung-up' movements, too slow for myoclonus, inhibitory rather than excitatory in some respects, and undeniably of central dysrhythmic origin as shown by corresponding EEG abnormalities. These attacks may be sufficiently distinctive to make a bedside diagnosis. Neurological abnormalities include a mixed pyramidal and striatal clinical picture.

If the diagnosis is uncertain clinically, the cistinctively periodic complexes in the EEG, a paritic Lange, and very high levels of measles antibody in both blood and CSF are usually enough to establish the diagnosis adequately without recourse to brain biopsy.

3. Cerebral palsy. It is well known that during the first year or two years of life certain forms of cerebral palsy, notably the diplegic and athetoid forms, have an evolving character. The circumstances in which these conditions occur normally help in differentiation: athetoid cerebral palsy usually follows either kernicterus (with or without prematurity) or birth asphyxia or shock, while diplegic cerebral palsy is usually seen as a consequence of prematurity in infants weighing less than $1.5 \mathrm{~kg}$. In their absence the clinician must be especially wary since a substantial proportion of the remainder include heredodegenerative syndromes.

4. Psychiatric problems. From time to time regression of an exceptionally severe degree proves very baffling to both psychiatrist and paediatrician. In the context of gross disturbance in the psychosocial environment-separation, deprivation, maltreatment, marital disharmony, psychopathy or psychosis in parents-and in the absence of evolving neurological abnormalities, it is most likely that psychological factors are pre-eminent, though it is virtually impossible to exclude an organic substrate at a given instant. Sensitive management of a grossly disturbed social situation usually allows reasonably confident clarification of the issues on clinical grounds alone, but the initial evaluation in the conventional setting of an anamnestic interview may be limited or misleading, and observation in the home by a trained observer may be much more illuminating. Mistakes will nevertheless be made occasionally, but perhaps it is better to manage overtly psychiatric problems which are relatively common, unfettered by fears of missing a progressive organic disease, than to be 
preoccupied about missing a rare (incurable) disease.

In so-called 'hysterical' illness in childhood, it is usually possible to identify reasonably easily the factors which either potentiate what may have originally begun as a trivial organic illness, or determine the form and content of the physical symptomatology.

During the ingravescent phase of autism, usually in the second year of life, there may be regression in speech and behaviour, without any physical manifestations of disease, but with behavioural changes characteristic of autism. It is unlikely to be a source of confusion more than very temporarily.

5. Epilepsy. Intellectual and neurological deterioration sometimes occurs in patients with idiopathic epilepsy. Both are not infrequently seen after status epilepticus and some patients may have, for example, evidence of a cerebellar deficit, transiently or permanently.

In some schoolchildren with epilepsy who may have neither particularly severe nor frequent fits there is reputedly a progressive lowering of IQ. On closer scrutiny of test results one usually finds that the child manages to complete more or less exactly what he achieved at his previous test, perhaps a year earlier. When this is related to age, of course it appears as a fall in IQ. One possible interpretation of this finding is that he has either stopped learning or is learning more slowly, and one suspects that this may be due as much to the depressant effects of anticonvulsant drugs, especially barbiturate and primidone, as to dysrhythmia. There is very rarely evidence of 'hard' neurological signs or degenerative disease.

In minor status epilepticus, by contrast, not only may there appear to be gross intellectual regression for periods lasting several months, but also recovery may be almost as dramatic once the dysrhythmia is controlled.

\section{Investigations}

Haematology. Haematological abnormalities are sometimes sufficiently distinctive to suggest a diagnosis. For example, a macrocytic anaemia may suggest an inborn abnormality of vitamin B12 metabolism, acquired abnormalities being very rare in childhood, while the presence of acanthocytes as evidence of a- $\beta$-lipoproteinaemia may give a simple and direct clue to the diagnosis of an obscure cerebellar syndrome.

Examination of the peripheral white cells may disclose significant numbers of vacuolated lympho- cytes as seen, for example, in Niemann-Pick disease, though it must be remembered that small numbers of abnormal-looking cells may be found in apparently normal individuals.

Marrow examination provides an extension of haematological studies especially valuable where there is a reticuloendothelial involvement. It may provide diagnostically significant material in, for example, Gaucher's disease, generalized $\mathrm{GM}_{1}$ gangliosidosis, and in 'sea blue histiocyte' disease (Lake, Stephens, and Neville, 1970). As a source of culture material for histochemical study it is rivalled by skin biopsy (see later).

Radiology. Diagnostically helpful radiological abnormalities of long bones and/or spine are seen in a variety of conditions where both marrow and matrix as well as epiphysial plate may be involved, e.g. the mucopolysaccharidoses, Gaucher's disease, mucolipidoses (Spranger and Wiedemann, 1970), homocystinuria, and the gangliosidoses.

Skull radiology can be most informative. Particularly helpful is the thickening and increase in diploic pattern with loss of 'digital' markings seen where there is cortical atrophy. Though this sign is seen after a single cerebral insult in childhood, after which the brain stops growing and there is critical shrinkage (as in reaction to pertussis antigen), it is often most prominent in the amaurotic family idiocies, particularly in some cases of longer duration, and represents in them progressive cortical atrophy.

Progressive calcification of Sturge-Weber disease beginning posteriorly and spreading forwards is often mistakenly thought to represent calcification in arterial walls because of its parallel and linear characteristics. The calcification seen on $x$-ray occurs in the gyri, and is in the substance of the cortex.

Contrast studies are not themselves diagnostic but may show localized, for example, cerebellar, or generalized, for example cortical, atrophy. The presence of the latter can, of course, be surmised if thickening of skull bones is seen on plain films of the skull. Air studies will serve to delineate aqueduct stenosis, posterior fossa, or brainstem tumours, or aneurysm of the great vein of Galen, conditions which, because of their progressive nature, may mimic heredodegenerative syndromes.

Electroencephalogram (EEG). At the same time as being most valuable in the diagnosis of certain progressive neurological diseases such as subacute sclerosing panencephalitis, late infantile (Bielschowsky) amaurotic idiocy, and giving other 
valuable nonspecific evidence of an evolving disease, the EEG may be most misleading, especially when seizure control is variable, showing wide and unsustained fluctuations in dysrhythmic pattern. The persisting lack of statistically controlled and standardized evaluation of EEG changes must still limit their value in this respect.

Of outstanding positive diagnostic value is the phenomenon described by Pampiglione (1961) and now validated in over a score of histologically proven cases of one of the late infantile forms of amaurotic family idiocy. It comprises an abnormal response to photic stimulation at low rates of flicker, with repetitive discharges at an idiosyncratic frequency independent of the higher flicker rates. This pathognomonic 'sign' has recently been 'rediscovered' (Green, 1971).

For departments with appropriate apparatus there is a computerized averaging technique for measuring cortically evoked responses to both auditory and visual stimuli. In the case of the latter the study of visually evoked responses in the occipital cortex is of special value in some of the degenerative diseases which specially affect optic radiations or occipital cortex, e.g. diffuse scleroses.

The study of visual pathways can be further clarified by measurement of the electroretinogram potentials, with an electrode placed on the bridge of the nose or on the corneal conjunctiva (Harden and Pampiglione, 1970). The electroretinogram response is diminished or absent in the tapetoretinal degenerations, and can be of particular help in investigation of the amaurotic family idiocies. In these conditions the response is lost at an early stage when ophthalmic signs may not be particularly obvious (Harcourt, 1970).

Electromyogram (EMG) and nerve conduction studies. The combination of EMG and nerve conduction studies has now achieved a degree of refinement and elaboration in young children as well as in adults to enable a reliable distinction not only between myopathic and neuropathic weakness but also valuable differentiation between segmental demyelination (e.g. metachromatic leucodystrophy, globoid cell leucodystrophy) and axonal degeneration (e.g. spinal muscular atrophy).

Biochemical studies. Certain biochemical investigations (especially those amenable to automatic analysis) are sufficiently cheap and reliable to be used extensively in screening studies of patients with fits or other neurological or mental handicaps. Nevertheless, in terms of the proportion of abnormal results found in this way, even allowing for a degree of selection of patients, the yield is quite low. Depending on the laboratory, certain tests can now be considered to be routine, e.g. urinary chromatography for amino acids and sugars; nitroprusside test on fresh urine (homocystinuria); urinary mucopolysaccharide screening tests; fasting blood sugar; uric acid; serum calcium; phosphate, alkaline, and acid phosphatases; and plasma cholesterol. Protein-bound iodine, magnesium, serum immunoglobulins (ataxia telangiectasia, dystrophia myotonica), pyruvate and lactate concentrations (Leigh's acute necrotizing infantile encephalomyelopathy), blood ammonia, are worth while considering as screening procedures; their expense can be justified by the fact that in several cases they can detect diseases which, however rare, may be amenable to treatment sooner or later, if not already. Their greater complexity requires first-class laboratory facilities with an acknowledged readiness to deal with paediatric problems, and where possible, with fingerprick samples of blood.

Other biochemical studies are deservedly restricted for conditions where clinical studies and preliminary screening have provided some selection of patients, in particular separating off cases of primary mental subnormality and cerebral palsy.

Likewise, assay of sphingomyelinase and $\beta$ glucocerebrosidase in white blood cells may establish, respectively, the biochemical basis of a diagnosis of Niemann-Pick (Kampine et al., 1967) or Gaucher's disease (Patrick, 1965). In $\mathrm{GM}_{1}$ gangliosidosis the various forms can be differentiated by white cell assays of the $\beta$-galactosidase isoenzymes ( $R$. Ellis, personal communication). Similarly isoenzyme assay of white cell and serum hexosaminidase is of value in differentiating the various forms of Tay-Sachs disease and the other $\mathrm{GM}_{2}$ gangliosidoses.

Although essential for a diagnosis of hepatolenticular degeneration, blood copper and copper oxidase are needlessly included in some neurological screening programmes since it is almost unknown for this condition to manifest itself as a neurological condition before the age of 10 years, though it may present as a haemolytic-hepatitic episode earlier.

The demonstration of an increased phytanic acid concentration in the blood is sufficient to establish a diagnosis of Refsum's disease, while plasma lipoprotein assay is necessary unequivocally to identify a- $\beta$-lipoproteinaemia (Bassen-Kornzweig disease) or an $\alpha$-lipoproteinaemia (Tangier disease), both of which have important neurological components.

Among the heterogeneous leucodystrophies, two main conditions have been separately identified: 
metachromatic leucodystrophy (late infantile, juvenile, and adult forms) and globoid cell leucodystrophy (Krabbe, or infantile form, and later forms).

Aryl sulphatase determinations in white blood cells and urine may clinch a diagnosis of metachromatic leucodystrophy, already strongly suggested by the clinical picture with evidence of slowing of nerve conduction, and by the demonstration of distinctive intracellular metachromatic material in fresh spun urinary sediment (Lake, 1965).

In Krabbe's leucodystrophy the enzyme galactocerebroside $\beta$-galactosidase is lacking (Austin et al., 1970), and this deficiency can be shown by an isotopic technique in white blood corpuscles.

Less well-defined biochemically among the diffuse scleroses is a sex-linked recessive form associated with suprarenal insufficiency (Siemerling and Creutzfeldt, 1923). Though the literature suggests that clinical adrenal insufficiency antedates the neurological disorder (Forsyth, Forbes, and Cumings, 1971), in my experience in a series of cases selected by their presentation as neurological syndromes (mimicking acute encephalopathies, or acute demyelinating diseases) the only evidence of suprarenal dysfunction has been reduced or absent cortisol production after ACTH stimulation.

\section{Biopsy}

Rectal mucosa and appendix. The purpose of a rectal biopsy is to obtain a full-thickness sample containing sufficient ganglion cells to allow histochemical study (Bodian and Lake, 1963). The staining may show intraneuronal lipid inclusions as in Tay-Sachs disease and the other gangliosidoses, or lipofuscin inclusions in the amaurotic family idiocies. In sulphatide lipidosis (metachromatic leucodystrophy) metachromatically-staining material is seen either lying apparently free, or phagocytosed in the ganglion-cell layer.

Though the histochemical demonstration of increased lysosomal acid phosphatase activity in the ganglion cells is nonspecific, its occurrence in an infant with a suggestive clinical picture makes a diagnosis of Krabbe's leucodystrophy very probable. If the technique of white cell assay of $\beta$-galactosidase mentioned above is not available, the diagnosis can only be established with certainty by brain biopsy.

A full-thickness biopsy carries with it a higher risk of haemorrhage, sepsis, and technical failure in inexperienced hands than removal of the appendix, and for these reasons the latter is often preferred. Ganglion cells are, however, smaller and abnormal staining correspondingly less impressive in the appendix than in the rectum (Brett and Berry, 1967).
At parents' request, histological examination of ganglion cells has been used at The Hospital for Sick Children on several occasions in clinically unaffected sibs of patients with either Tay-Sachs disease or late infantile amaurotic idiocy to make either a premorbid diagnosis of the same condition or to establish the normality of the child.

Brain biopsy. The amounts of neural tissue obtained by rectal biopsy or appendicectomy are insufficient for even semiquantitative chemical analysis, and allow only limited histochemical and ultrastructural study. To obtain sufficient grey and white matter for these purposes and for enzyme studies, a brain biopsy is essential if there is strong evidence to suppose that there is diffuse cortical involvement.

The drawbacks are obvious: there is a significant morbidity, if not mortality, even in the most skilled hands; the amount of tissue removed is relatively small; it is difficult to be sure in advance that cortical involvement is sufficiently diffuse to yield a specifically abnormal abnormality in the few 'silent' sites available for brain biopsy. If it is concluded on clinical grounds that the degenerative process is predominantly striatal or brainstem in situation, such as in Hallevorden-Spatz disease and olivopontocerebellar degeneration, or if it is likely that neuropathological characterization depends on appraisal of the total distribution of cerebral lesions, cortical biopsy is useless.

The main clinical prerequisites for selection of patients are dementia, and clinical and preferably EEG evidence of a diffuse cortical process.

The number of occasions on which a diagnosis can be achieved in life by brain biopsy alone are very rare and dwindling since the commonest degenerative 'storage' diseases seen in the U.K., i.e. Tay-Sachs, amaurotic family idiocy, and metachromatic leucodystrophy, can be diagnosed on other evidence. The only current example of brain biopsy which can be justified for potential therapeutic reasons is herpetic encephalitis; the justification in the degenerative diseases is prognostic and genetic.

It is appropriate here to acknowledge the need for careful morbid anatomical studies in all children dying of obscure neurological disease. Not only is this required as a sobering corrective for supposed clinical expertise, but also especially for accurate genetic counselling. Since this is of tremendous importance to parents who are usually still in their procreative years, and, having recently lost a child, may be especially anxious to extend their family, the need for detailed neuropathological studies 
assumes an urgency which many pathologists are slow to realize.

Tissue culture. A number of specialized laboratories are concentrating efforts on developing tissue culture techniques by which both the abnormally stored material and the enzymic defect can be identified in fibroblast culture (Sloan, 1970). This is because the metabolic abnormalities underlying many of the neurolipidoses are not restricted only to neuraxial tissue.

Such studies are of interest at present mainly for research purposes, for example in the mucopolysaccharidoses (Wiesmann and Neufeld, 1970), the mucolipidoses (Spranger and Wiedemann, 1970; Leroy et al., 1971; Tondeur et al., 1971), TaySachs disease (Schneck et al., 1970), $\mathrm{GM}_{1}$ gangliosidosis (Sloan et al., 1969), and metachromatic leucodystrophy (Porter et al., 1970), but the technique will undoubtedly be of great practical importance.

Enzymological and histochemical studies in cultured tissue is of special value in those conditions in which amniotic cells either normally contain a particular enzyme or accumulate an identifiable storage compound when abnormal. This technique has obvious application in prenatal diagnosis and allows 'positive' genetic counselling in a steadily increasing number of conditions, of which one might quote Tay-Sachs disease (Schneck et al., 1970) and metachromatic leucodystrophy (Porter et al., 1970).

Peripheral nerve biopsy. Segmental demyelination of peripheral nerves is well recognized in metachromatic leucodystrophy (Fullerton, 1964) and in globoid cell leucodystrophy (Lake, 1968; Dunn et al., 1969). This may be suspected not only from the clinical picture but also from the slowing of nerve conduction, and a specimen suitable for both histochemical and ultrastructural study can be obtained just behind the lateral malleolus. In metachromatic leucodystrophy the characteristic staining reaction can usually be shown easily if there is evidence of electrical abnormality.

In hypertrophic interstitial neuritis, the distinctive onion-skin appearance will identify the disease before clinical evidence of nerve hypertrophy is discovered, but the condition can be surmised without biopsy in Refsum's disease, if increased phytanic acid concentration is shown in plasma.

In summing up it is acknowledged that, individually, the conditions mentioned here are rare, and even as a group they represent a small minority of the neurological and neuropsychiatric problems in paediatric practice. But since they often present and masquerade as more benign conditions such as cerebral palsy or epilepsy, the paediatrician's perturbation about not missing a sinister disease may tempt him into the indiscriminate use of procedures that are unnecessary. From the array of biochemical abnormalities now identified and the welter of laboratory studies available, the clinician may be forgiven some bewilderment. It is suggested that the use of both potentially harmful as well as complex and expensive investigations must be concentrated in appropriately equipped and specialized units.

\section{REFERENCES}

Austin, J., Suzuki, K., Armstrong, D., Brady, R., Bachhawat, B. K., Schlenker, J., and Stumpf, D. (1970). Studies in globoid (Krabbe) leukodystrophy (GLD). V. Controlled enzymic studies in ten human cases. Archives of Neurology, 23, 502.

Bodian, M., and Lake, B. D. (1963). The rectal approach to neuropathology. British fournal of Surgery, 50, 702.

Brett, E. M., and Berry, C. L. (1967). Value of rectal biopsy in paediatric neurology: report of 165 biopsies. British Medical fournal, 3, 400.

Dunn, H. G., Lake, B. D., Dolman, C. L., and Wilson, J. (1969). The neuropathy of Krabbe's infantile cerebral sclerosis (globoid cell leucodystrophy). Brain, 92, 329.

Forsyth, C. C., Forbes, M., and Cumings, J. N. (1971). Adrenocortical atrophy and diffuse cerebral sclerosis. Archives of Disease in Childhood, 46, 273.

Fullerton, P. M. (1964). Peripheral nerve conduction in metachromatic leucodystrophy (sulphatide lipidosis). fournal of Neurology, Neurosurgery and Psychiatry, 27, 100.

Green, J. B. (1971). Neurophysiological studies in Batten's disease. Developmental Medicine and Child Neurology, 13, 477.

Harcourt, B. (1970). Electroretinography and the diagnosis of tapeto-retinal degeneration in childhood. Developmental Medicine and Child Neurology, 12, 775.

Harden, A., and Pampiglione, G. (1970). Neurophysiological approach to disorders of vision. Lancet, $1,805$.

Kampine, J. P., Brady, R. O., Kanfer, J. N., Feld, M., and Shapiro, D. (1967). Diagnosis of Gaucher's disease and Niemann-Pick disease with small samples of venous blood. Science, 155, 86.

Lake, B. D. (1965). A reliable, rapid screening test for sulphatide lipidosis. Archives of Disease in Childhood, 40, 284.

Lake, B. D. (1968). Segmental demyelination of peripheral nerves in Krabbe's disease. Nature (London), 217, 171.

Lake, B. D., Stephens, R., and Neville, B. G. R. (1970). Syndrome of the 'sea-blue histiocyte'. Lancet, $2,309$.

Leroy, J. G., Spranger, J. W., Feingold, M., Opitz, J. M., and Crocker, A. C. (1971). I-cell disease: a clinical picture. fournal of Pediatrics, 79, 360.

Pampiglione, G. (1961). E.E.G. in some inborn errors of metabolism. VIIth International Congress of Neurology, Rome, September 1961, Vol. 1, p. 53. Soc. Graf. Romana, Rome. Also abst. in Excerpta med. int. Congr. Ser., 39, 2.

Patrick, A. D. (1965). A deficiency of glucocerebrosidase in Gaucher's disease. Biochemical fournal, 97, 17c.

Porter, M. T., Fluharty, A. L., Harris, S. E., and Kihara, H. (1970). The accumulation of cerebroside sulfates by fibroblasts in culture from patients with late infantile metachromatic leukodystrophy. Archives of Biochemistry and Biophysics, 138, 646.

Schneck, L., Friedland, J., Valenti, C., Adachi, M., Amsterdam, D., and Volk, B. W. (1970). Prenatal diagnosis of Tay-Sachs disease. Lancet, 1, 582.

Siemerling, E., and Creutzfeldt, H. G. (1923). Bronzekrankheit und sklerosierende Encephalomyelitis. Archiv für Psychiatrie und Nervenkrankheiten, 68, 217. 
Sloan, H. R. (1970). Tissue culture studies in the lipid storage disorders. Chemistry and Physics of Lipids, 5, 250.

Sloan, H. R., Uhlendorf, B. W., Jacobson, C. B., and Fredrickson, D. S. (1969). $\beta$-galactosidase in tissue culture derived from human skin and bone marrow: enzyme defect in $\mathrm{GM}_{1}$ gangliosidosis. Pediatric Research, 3, 532.

Spranger, J. W., and Wiedemann, H. R. (1970). The genetic mucolipidoses. Neuropädiatrie, 2, 3.
Tondeur, M., Vamos-Hurwitz, E., Mockel-Pohl, S., Dereume, J. P., Cremer, N., and Loeb, H. (1971). Clinical, biochemical, $\overline{\bar{O}}$ and ultrastructural studies in a case of chondrodystrophy presenting the I-cell phenotype in tissue culture. fournal of Pediatrics, 79, 366.

Wiesmann, U., and Neufeld, E. F. (1970). Scheie and Hurler syndromes: apparent identity of the biochemical defect. Science, 169, 72. 